\title{
Disorder Dependence of Interface Spin Memory Loss
}

\author{
Kriti Gupta $\odot,{ }^{1}$ Rien J. H. Wesselink, ${ }^{1}$ Ruixi Liu, ${ }^{2}$ Zhe Yuan $\odot,{ }^{2, *}$ and Paul J. Kelly $\oplus^{1,2, \dagger}$ \\ ${ }^{1}$ Faculty of Science and Technology and MESA ${ }^{+}$Institute for Nanotechnology, University of Twente, \\ P.O. Box 217, 7500 AE Enschede, The Netherlands \\ ${ }^{2}$ The Center for Advanced Quantum Studies and Department of Physics, Beijing Normal University, 100875 Beijing, China
}

(Received 11 November 2019; revised manuscript received 17 January 2020; accepted 29 January 2020; published 27 February 2020)

\begin{abstract}
The discontinuity of a spin-current through an interface caused by spin-orbit coupling is characterized by the spin memory loss (SML) parameter $\delta$. We use first-principles scattering theory and a recently developed local current scheme to study the SML for $\mathrm{Au}|\mathrm{Pt}, \mathrm{Au}| \mathrm{Pd}, \mathrm{Py} \mid \mathrm{Pt}$, and $\mathrm{Co} \mid \mathrm{Pt}$ interfaces. We find a minimal temperature dependence for nonmagnetic interfaces and a strong dependence for interfaces involving ferromagnets that we attribute to the spin disorder. The SML is larger for Co|Pt than for Py|Pt because the interface is more abrupt. Lattice mismatch and interface alloying strongly enhance the SML that is larger for a $\mathrm{Au} \mid \mathrm{Pt}$ than for a $\mathrm{Au} \mid \mathrm{Pd}$ interface. The effect of the proximity-induced magnetization of $\mathrm{Pt}$ is negligible.
\end{abstract}

DOI: 10.1103/PhysRevLett.124.087702

With the discovery of the giant magnetoresistance effect in magnetic multilayers [1,2], it was recognized that interfaces play a key role in spin transport phenomena. In semiclassical formulations [3-5] of transport, these appear as discrete resistances and the description of the transport of electrons through a multilayer requires a resistivity $\rho$ for each material as well as a resistance $R_{\mathrm{I}}$ for each interface. Bulk resistivities are readily measured, interface resistances much less so. Magnetic materials require spin-dependent bulk resistivities $\rho_{\sigma}$ and interface resistances $R_{\sigma}$. Because spin is not conserved, describing its transport additionally requires a spin-flip diffusion length (SDL) $l_{\text {sf }}$ in each material, as well as its counterpart for each interface, the spin memory loss (SML) parameter $\delta$. Determining $l_{\mathrm{sf}}$ requires distinguishing interface and bulk contributions. Because doing so is nontrivial, the interface contribution had been largely neglected and values of $l_{\mathrm{sf}}$ reported over the last decade for well-studied materials like Pt span an order of magnitude [6-8].

Almost everything we know about interface parameters is from current-perpendicular-to-the-plane (CPP) magnetoresistance experiments $[5,6,9]$ interpreted using the semiclassical Valet-Fert (VF) model [4]. Though these experiments are relatively simple to interpret, they are restricted to low temperatures as they require superconducting leads [5], while calculations have so far only addressed ballistic interfaces [10-15]. Because the vast majority of experimental studies in spintronics is carried out at room temperature, there is a need to know how transport parameters, in particular, those describing interfaces, behave at finite temperatures.

This need is accentuated by the huge interest in recent years $[7,16]$ in the spin Hall effect (SHE) [17-19], whereby a longitudinal charge current drives a transverse spin current in nonmagnetic materials, and in its inverse, the inverse SHE (ISHE). Determination of the spin Hall angle (SHA) $\Theta_{\mathrm{sH}}$ that measures the efficiency of the SHE is intimately connected with the SDL and, because an interface is always involved, with the SML [20]. When use is made of spin pumping and the ISHE [21-24] or the SHE and spintransfer torque [25], the interface in question is between ferromagnetic (FM) and nonmagnetic (NM) materials. When the nonlocal spin-injection method is used [26,27], two interfaces are involved: an $\mathrm{FM} \mid \mathrm{NM}$ interface to create a spin accumulation and an $\mathrm{NM} \mid \mathrm{NM}^{\prime}$ interface to detect it. Progress has been made by recognizing that bulk parameters like $l_{\mathrm{sf}}$ and $\Theta_{\mathrm{sH}}$ are very sample dependent and that the SML plays a key role in determining their values [20,28-30]. Recent studies suggesting that measurements of the SHA may actually be dominated by interface effects [20,28,31,32] are stimulating attempts to tailor these [33-37].

This makes it crucial to have a way to independently determine interface parameters. We recently described a formalism to evaluate local charge and spin currents [8] from the solutions of fully relativistic quantum mechanical scattering calculations [38] that include temperatureinduced lattice and spin disorder [39,40]. This yielded a layer-resolved description of spin currents propagating through atomic layers of thermally disordered Pt and Py that allowed us to unambiguously determine bulk transport properties [41]. By focusing on spin currents, we can straightforwardly evaluate all the parameters entering the Valet-Fert semiclassical formalism [4] that is universally used to interpret experiment [5].

In this Letter, we focus on interface transport properties and study realistic interfaces between thermally disordered materials. Typical structures used in experimental studies of the SHE contain a heavy NM metal with strong spin-orbit 
coupling (SOC) and a $3 d$ transition metal (TM) or TM alloy ferromagnet [7,20-25,42]. We will study (i) $\mathrm{Au} \mid \mathrm{Pt}$ and $\mathrm{Au} \mid \mathrm{Pd}$ interfaces to shed light on the role of SOC and roughness at interfaces involving heavy TMs and (ii) $\mathrm{Py} \mid \mathrm{Pt}$ and $\mathrm{Co} \mid \mathrm{Pt}$ interfaces to examine the role of the FM magnetization and disorder in determining interface parameters, as well as the temperature dependence of all these.

Method.-We begin by solving the VF equations analytically for the spin accumulation $\mu_{s i}(z)$ and spin current $j_{s i}(z)$ in a metallic multilayer. The solution in each region $i$ involves two coefficients $A_{i}$ and $B_{i}$ that are determined by appropriate boundary conditions [4]. For an $\mathrm{NM} \mid \mathrm{NM}^{\prime}$ system, we will require that $j_{s}(0)=1$ at the left-lead|NM interface, corresponding to injecting a fully spin polarized current from the left lead, and that $j_{s}(\infty)=0$, requiring the $\mathrm{NM}^{\prime}$ material to be much thicker than its $l_{\mathrm{sf}}$ value. The interface (I) is initially considered as a bulklike material with resistivity $\rho_{\mathrm{I}}, \operatorname{SDL} l_{\mathrm{I}} \equiv l_{\text {sf }}^{\mathrm{I}}$, and thickness $t$ so that at the $\mathrm{NM} \mid \mathrm{I}$ and $\mathrm{I} \mid \mathrm{NM}^{\prime}$ interfaces the spin accumulation and spin current are continuous $[43,44]$. We then eliminate the coefficients $A_{\mathrm{I}}$ and $B_{\mathrm{I}}$ and take the limit $t \rightarrow 0$, thereby defining the areal interface resistance $A R_{\mathrm{I}}$ and the SML parameter $\delta$ as

$$
A R_{\mathrm{I}}=\lim _{t \rightarrow 0} \rho_{\mathrm{I}} t \quad \text { and } \quad \delta=\lim _{t \rightarrow 0} t / l_{\mathrm{I}}
$$

We finally express $\delta$ as

$$
\frac{j_{s, \mathrm{NM}}\left(z_{\mathrm{I}}\right)}{j_{s, \mathrm{NM}^{\prime}}\left(z_{\mathrm{I}}\right)}=\cosh \delta+\frac{\rho_{\mathrm{NM}^{\prime}} l_{\mathrm{NM}^{\prime}}}{A R_{\mathrm{I}}} \delta \sinh \delta
$$

in terms of $j_{s i}\left(z_{\mathrm{I}}\right)$, the spin current at the interface $z_{\mathrm{I}}$ on the $i=\mathrm{NM}$ and $\mathrm{NM}^{\prime}$ sides as well as $\rho_{\mathrm{NM}^{\prime}}, l_{\mathrm{NM}^{\prime}} \equiv l_{\mathrm{sf}}^{\mathrm{NM}^{\prime}}$, and $R_{\mathrm{I}}$. The relationship of the SML to the spin-current discontinuity $j_{s, \mathrm{NM}}\left(z_{\mathrm{I}}\right)-j_{s, \mathrm{NM}^{\prime}}\left(z_{\mathrm{I}}\right)$ is nontrivial.

$\mathrm{Au} \mid \mathrm{Pt}$ interface.-We illustrate our methodology in Fig. 1 for a $\mathrm{Au} \mid \mathrm{Pt}$ interface between "room-temperature" $\mathrm{Au}$ and $\mathrm{Pt}$, in which a Gaussian distribution of atomic displacements in a $7 \times 7$ lateral supercell was used to reproduce the experimentally observed resistivities of each bulk material at $T=300 \mathrm{~K}, \rho_{\mathrm{Au}}=2.3 \mu \Omega \mathrm{cm}$ and $\rho_{\mathrm{Pt}}=$ $10.7 \mu \Omega \mathrm{cm}$ [45], for which $l_{\mathrm{Au}} \sim 80 \mathrm{~nm}$ and $l_{\mathrm{Pt}} \sim 5.25 \pm$ $0.05 \mathrm{~nm}$ [8]. The empty gray circles in Fig. 1 represent $j_{s}(z)$, obtained [46] from the results of quantum mechanical scattering calculations [38] for a $\mathrm{Au} \mid \mathrm{Pt}$ bilayer when a fully polarized spin current was injected into the bilayer from the left $\mathrm{Au}$ lead. The lattice constant of fcc $\mathrm{Au}$ is initially chosen to be that of $\mathrm{Pt}\left(a_{\mathrm{Au}}^{\prime}=a_{\mathrm{Pt}}=3.923 \AA\right)$, which does not affect the Au electronic structure qualitatively. The figure also shows the VF solutions in $\mathrm{Au}$ (blue curve) and Pt (red curve) found by fitting $j_{s}(z)$ far from the interface. The initial spatial decay of $j_{s}(z)$ is determined by $l_{\mathrm{Au}}$, the rapid decay in the vicinity of the interface is described in the semiclassical VF framework by the interface discontinuity and, after this abrupt decay, the spin

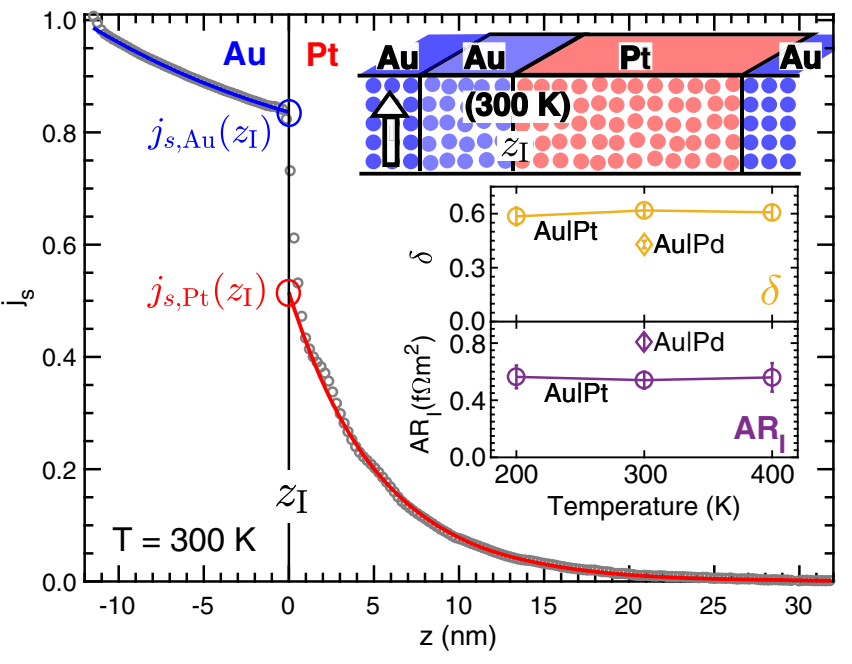

FIG. 1. A fully polarized spin-current $j_{s}$ injected at $300 \mathrm{~K}$ from the left $\mathrm{Au}$ lead into a $\mathrm{Au}(50) \mid \operatorname{Pt}(140)$ bilayer sandwiched between $\mathrm{Au}$ leads decays exponentially in $\mathrm{Au}$ and in $\mathrm{Pt}$; the numbers in brackets denote the number of atomic layers. The solid lines indicate fits for $j_{s}(z)$ in individual layers using solutions of the VF equations. (Top inset) Schematic of the scattering region. (Middle and bottom insets) Temperature dependence of the interface parameters $\delta$ (yellow) and $R_{\mathrm{I}}$ (purple) for a $\mathrm{Au} \mid \mathrm{Pt}$ interface (circles). The corresponding parameters for $\mathrm{Au} \mid \mathrm{Pd}$ at $300 \mathrm{~K}$ are included (diamonds).

current that survives in Pt decays to zero on a length scale described by $l_{\mathrm{Pt}}$.

By fitting $j_{s}(z)$ to the solution of the VF equation, we obtain values of $j_{s, \mathrm{Au}}\left(z_{\mathrm{I}}\right)$ and $j_{s, \mathrm{Pt}}\left(z_{\mathrm{I}}\right)$. From the Landauer expression for the conductance in terms of the transmission matrices, $A R_{\mathrm{I}}=0.54 \pm 0.03 \mathrm{f} \Omega \mathrm{m}^{2}$ is directly determined, leaving just $\delta$ as the only unknown parameter. Using a numerical root finder to solve (2), we find $\delta=0.62 \pm 0.03$, where the error bar is evaluated from the uncertainties in the other parameters.

The bulk parameters $\rho_{\mathrm{Pt}}$ and $1 / l_{\mathrm{Pt}}$ are known to increase linearly with temperature [31,40,45,47], but virtually nothing is known about the temperature dependence of interface parameters. We therefore calculate $A R_{\mathrm{Au} \mid \mathrm{Pt}}$ and $\delta$ at 200 and $400 \mathrm{~K}$ and plot the results in Fig. 1 (inset). Within the error bars of the calculations, both parameters remain constant between 200 and $400 \mathrm{~K}$. The temperature independence that we observe for $\delta$ is in agreement with the results of a very recent CPP-magnetoresistance experiment for a $\mathrm{Cu} \mid \mathrm{Pt}$ interface that shows $\delta$ to be nearly constant over the temperature range 0-300 $\mathrm{K}$ [48].

Interface mixing.-Unlike the sharp interfaces between bulk $\mathrm{Au}$ and $\mathrm{Pt}$ we have considered so far, experimental interfaces are believed to comprise a few intermixed layers. To study the effect of interface mixing, we insert $N$ atomic layers of $\mathrm{Au}_{50} \mathrm{Pt}_{50}$ random alloy at the interface of the lattice-matched $\mathrm{Au} \mid \mathrm{Pt}$ bilayer [49]. The results for the spin current $j_{s}(z)$ and corresponding values of $\delta$ at $300 \mathrm{~K}$ are 


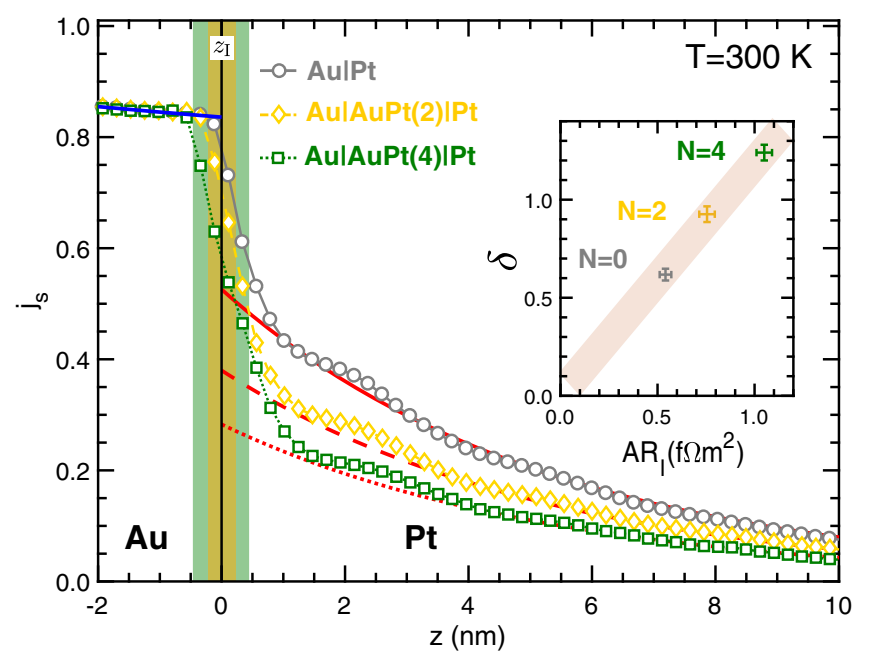

FIG. 2. A fully polarized spin current $j_{s}(z)$ is injected into a $\mathrm{Au} \mid \mathrm{Pt}$ bilayer with a sharp interface (vertical black line), two layers of $\mathrm{Au}_{50} \mathrm{Pt}_{50}$ interface (yellow shaded region), and four layers of $\mathrm{Au}_{50} \mathrm{Pt}_{50}$ interface (green shaded region) between them. The calculated spin currents $j_{s}(z)$ for the three cases are shown as gray circles, yellow diamonds, and green squares, respectively. The solid blue line indicates a fit to the VF equation in Au. The solid, dashed, and dotted red lines indicate fits to the VF equation in $\mathrm{Pt}$ for $\mathrm{Au}|\mathrm{Pt}, \mathrm{Au}| \mathrm{Au}_{50} \mathrm{Pt}_{50}(2) \mid \mathrm{Pt}$, and $\mathrm{Au}\left|\mathrm{Au}_{50} \mathrm{Pt}_{50}(4)\right| \mathrm{Pt}$, respectively. (Inset) $\delta$ vs $A R_{\mathrm{I}}$ for $N=0,2$, and 4 interface layers of mixed $\mathrm{Au}_{50} \mathrm{Pt}_{50}$.

shown in Fig. 2 for $N=0,2$, and 4. When the spin current from bulk $\mathrm{Au}$ approaches the mixed interface layers (yellow for $N=2$, green for $N=4$ ), then compared to the sharp interface, $j_{s}(z)$ decreases more and $\delta$ increases rapidly with increasing $N$ (inset). Electron scattering at a commensurable and clean $\mathrm{Au} \mid \mathrm{Pt}$ interface only involves Bloch states with equal $\mathbf{k}_{\|}$, but intermixing (and thermal disorder) break momentum conservation and allow $\mathbf{k}_{\|} \rightarrow \mathbf{k}_{\|}^{\prime}$ scattering. The higher scattering rate results in a higher spin-flipping probability and hence a larger $\delta$ for the intermixed interfaces. Moreover, conduction electrons injected into $\mathrm{Au}$ are only weakly affected by SOC until they enter $\mathrm{Pt}$, where as $d$ states they become very susceptible to the large SOC. The interatomic mixing effectively increases the region where conduction electrons experience large SOC and therefore increases the SML. The interface resistance $A R_{\mathrm{I}}$ also increases monotonically as the disordered region increases in thickness, suggesting that $\delta \propto A R_{\mathrm{I}}$, so $\rho_{\mathrm{I}} l_{\mathrm{I}} \sim$ const.

Lattice mismatch.-To study the effect of $\mathbf{k}_{\|} \rightarrow \mathbf{k}_{\|}^{\prime}$ scattering on its own, we reexamine the sharp (111) $\mathrm{Au} \mid \mathrm{Pt}$ interface where both $\mathrm{Au}$ and $\mathrm{Pt}$ have their equilibrium bulk volumes, $a_{\mathrm{Au}}=4.078$ and $a_{\mathrm{Pt}}=3.923 \AA$. A (111) oriented $5 \times 5$ unit cell of $\mathrm{Au}$ matches to a (111) oriented $3 \sqrt{3} \times 3 \sqrt{3}$ unit cell of Pt to better than $0.02 \%$. For this fully relaxed $\mathrm{Au} \mid \mathrm{Pt}$ geometry, we repeat our calculations at $300 \mathrm{~K}$ and obtain $\delta=0.81 \pm 0.05$ compared to $\delta=0.62 \pm 0.03$ with commensurable Au. This calculation indicates that the $\mathbf{k}_{\|} \rightarrow \mathbf{k}_{\|}^{\prime}$ scattering does indeed lead to an increase of the interface SML. Our finding is in agreement with calculations for a $\mathrm{Cu} \mid \mathrm{Pd}$ interface using the ansatz of Schep et al. [10,50], which indicated that $\delta$ increases on going from a sharp to a rough interface [14].

$\mathrm{Au} \mid \mathrm{Pd}$.- To examine the effect of changing the strength of the SOC, we apply the procedures described above to a commensurable $\mathrm{Au} \mid \mathrm{Pd}$ interface, choosing $a_{\mathrm{Au}}^{\prime}=a_{\mathrm{Pd}}=$ $3.891 \AA$. Corresponding to the experimental resistivity of $\mathrm{Pd}$ at $300 \mathrm{~K}, \rho_{\mathrm{Pd}}=10.8 \mu \Omega \mathrm{cm}[45]$, we find $l_{\mathrm{Pd}}=7.06 \pm$ $0.02 \mathrm{~nm}$ and a value of $A R_{\mathrm{Au} \mid \mathrm{Pd}}=0.81 \pm 0.05 \mathrm{f} \Omega \mathrm{m}^{2}$, which is much larger than the value $0.54 \pm 0.03 \mathrm{f} \Omega \mathrm{m}^{2}$ found for $\mathrm{Au} \mid \mathrm{Pt}$ (inset Fig. 1, bottom panel). By substituting all the input parameters and their uncertainties into (2), we extract a value of $\delta_{\mathrm{Au} \mid \mathrm{Pd}}=0.43 \pm 0.02$ (Fig. 1, inset). Compared to $\mathrm{Au} \mid \mathrm{Pd}$, the larger SOC in Pt leads to a larger value of $\delta$ for $\mathrm{Au} \mid \mathrm{Pt}$. Our results for $A R_{\mathrm{I}}$ and $\delta$ for $\mathrm{Au} \mid \mathrm{Pd}$ interfaces are in good agreement with theoretical estimates made by Belashchenko et al. [14], combining the ansatz of Schep et al. [10] with calculations for ballistic $\mathrm{Cu} \mid \mathrm{Pd}$ interfaces. $\mathrm{Cu}$ and $\mathrm{Au}$ have very similar electronic structures and the very different SOC of the filled $3 d$ and $5 d$ states below the Fermi level is not expected to play a major role.

$\mathrm{FM} \mid \mathrm{Pt}$ interfaces.-We developed an analogous procedure to study $\mathrm{FM} \mid \mathrm{NM}$ interfaces. Compared to the $\mathrm{NM} \mid \mathrm{NM}^{\prime}$ case, two additional parameters enter: spin asymmetry parameters $\beta=\left(\rho_{\downarrow}-\rho_{\uparrow}\right) /\left(\rho_{\downarrow}+\rho_{\uparrow}\right)$ for the bulk FM and $\gamma=\left(R_{\downarrow}-R_{\uparrow}\right) /\left(R_{\downarrow}+R_{\uparrow}\right)$ for the interface. To avoid interfaces between a lead and Py or Co, we considered symmetric NM|FM|NM scattering geometries and studied them by passing an unpolarized charge current through them. The appropriate boundary conditions are that both the spin accumulation and spin current vanish at $z= \pm \infty$ and the analysis results in two implicit equations, containing the discontinuity in the spin current at the $\mathrm{FM} \mid \mathrm{NM}$ interface, as described by $j_{s, \mathrm{FM}}\left(z_{I}\right)$ and $j_{s, \mathrm{Pt}}\left(z_{\mathrm{I}}\right)$, as well as the eight transport parameters $\rho_{\mathrm{NM}}, l_{\mathrm{sf}}^{\mathrm{NM}}, \rho_{\mathrm{FM}}, l_{\mathrm{sf}}^{\mathrm{FM}}, \beta_{\mathrm{FM}}, R_{\mathrm{I}}, \delta$, and $\gamma$. Figure 3(a) illustrates the spin current $j_{s}(z)$ that we calculate for a Pt $|\mathrm{Py}| \mathrm{Pt}$ trilayer at $300 \mathrm{~K}$. The five bulk parameters are determined independently as well as $A R_{\mathrm{I}}$ obtained from the Landauer formula, leaving us with two equations and two unknowns, $\delta$ and $\gamma$, to be determined.

This procedure was applied to $\mathrm{Py} \mid \mathrm{Pt}$ and $\mathrm{Co} \mid \mathrm{Pt}$ interfaces assuming completely relaxed geometries and $8 \times 8$ interface unit cells of (111) Py or Co matched to $2 \sqrt{13} \times 2 \sqrt{13}$ interface unit cells of Pt. Thermal lattice and spin disorder were taken into account as described in Refs. [8,38-40]. $\rho_{\mathrm{Pt}}$ and $l_{\mathrm{Pt}}$ were already determined above and the appropriate corresponding calculations were performed for bulk Py and Co $[40,51]$.

The temperature dependence of the three interface parameters that we extract for $\mathrm{Py} \mid \mathrm{Pt}$ and $\mathrm{Co} \mid \mathrm{Pt}$ interfaces 

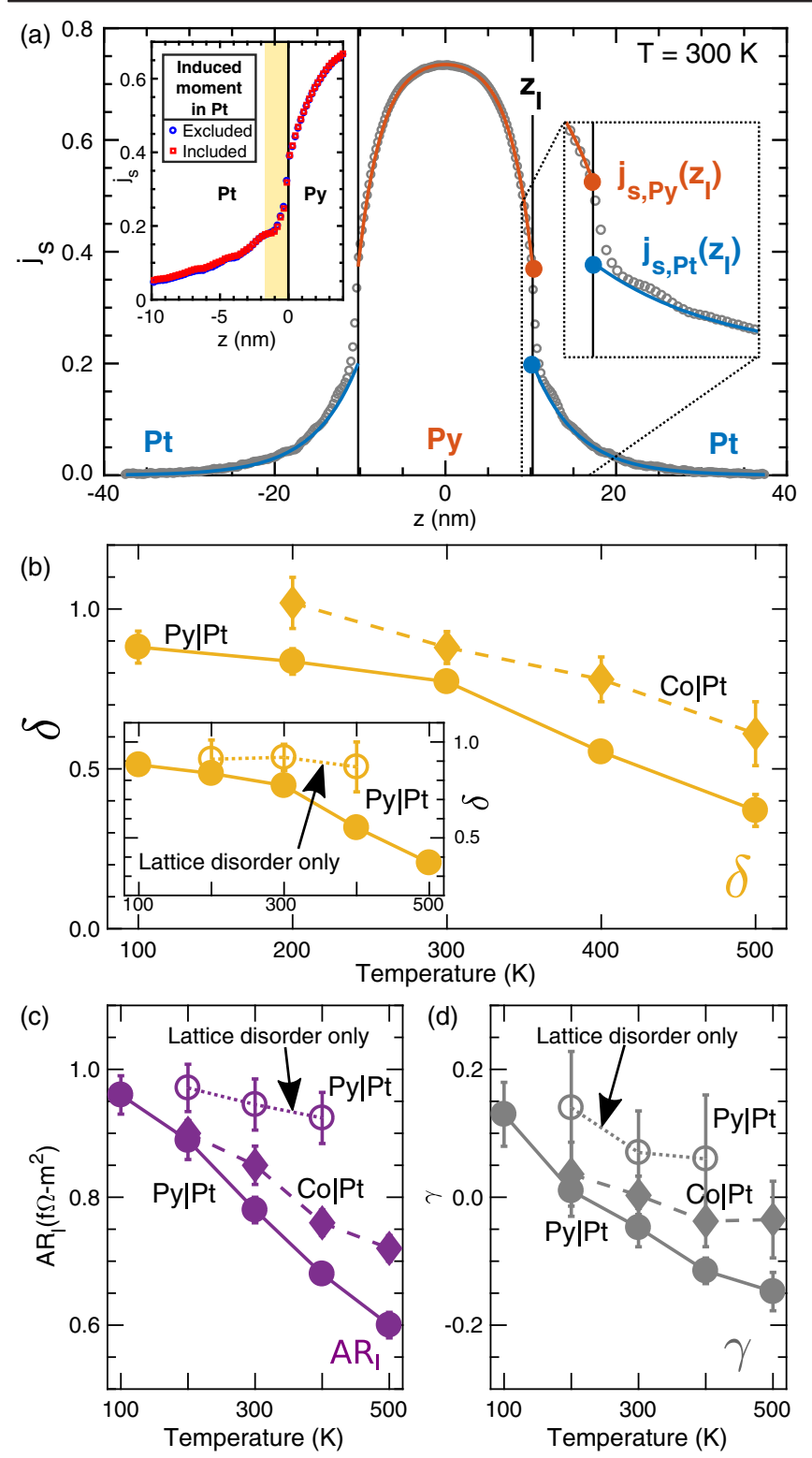

FIG. 3. (a) Open circles: spin current $j_{s}(z)$ through a Pt $|\mathrm{Py}| \mathrm{Pt}$ trilayer calculated for $T=300 \mathrm{~K}$. The solid blue (orange) curve is a fit to the VF equations in bulk Pt (Py). These fits are extrapolated to the interface $z_{\mathrm{I}}$ to obtain the values $j_{s, \mathrm{Pt}}\left(z_{\mathrm{I}}\right)$ and $j_{s, \mathrm{Py}}\left(z_{\mathrm{I}}\right)$, shown in detail in the right inset. (Left inset) The spin current with (red) and without (blue) proximity-induced moments in Pt. (b) $\delta$ for $\mathrm{Py} \mid \mathrm{Pt}$ (circles, solid lines) and $\mathrm{Co} \mid \mathrm{Pt}$ (diamonds, dashed lines) plotted as a function of temperature. (Inset) $\delta$ for $\mathrm{Py} \mid \mathrm{Pt}$ compared with results with only lattice disorder in $\mathrm{Py} \mid \mathrm{Pt}$ (open circles, dotted lines). (c),(d) Interface parameters $A R_{\mathrm{I}}$ and $\gamma$ for Py $\mid \mathrm{Pt}$ (circles, solid and dotted lines) and $\mathrm{Co} \mid \mathrm{Pt}$ (diamonds, dashed lines) plotted as a function of temperature. The dotted lines show the results for $\mathrm{Py} \mid \mathrm{Pt}$ with only lattice disorder.

is summarized in Figs. 3(b)-3(d). $A R_{\mathrm{I}}$ and $\gamma$ are seen to decrease monotonically with temperature for both interfaces. $\gamma$ is found to vary in a small range of \pm 0.15 for $\mathrm{Py} \mid \mathrm{Pt}$ and \pm 0.03 for $\mathrm{Co} \mid \mathrm{Pt} . \delta$, the main focus of our interest, decreases monotonically and substantially with temperature for both interfaces. $\delta_{\mathrm{Co} \mid \mathrm{Pt}}$ is larger than $\delta_{\mathrm{Py} \mid \mathrm{Pt}}$ for all temperatures in the range $200-500 \mathrm{~K}$. This temperature dependence contrasts starkly with the temperature independence we found for $\mathrm{Au} \mid \mathrm{Pt}$. We speculate that it is the variation of the spin disorder associated with the FM magnetization that affects the interface parameters most. Consistent with this is our finding that $\delta$ and $A R_{\mathrm{I}}$ are larger for $\mathrm{Co} \mid \mathrm{Pt}$ than for Py $\mid \mathrm{Pt}$. With a higher Curie temperature, $\mathrm{Co}$ is more ordered at any given temperature than Py.

To test this hypothesis, we repeated the $T=200,300$, and $400 \mathrm{~K}$ calculations for Py|Pt keeping the atomic spins ordered and including only lattice disorder in Py. The results for the three interface parameters with only lattice disorder are included in Fig. 3 (open circles, dotted lines) for comparison. With only lattice disorder included, we find that the $\mathrm{Py} \mid \mathrm{Pt}$ interface parameters decrease much more slowly with temperature. This weak variation can be attributed to the lattice disorder, but the decrease is much smaller compared to that brought about by spin disorder. In the low-temperature limit, we also expect $\delta$ to be smaller for $\mathrm{Py} \mid \mathrm{Pt}$ because this interface is less abrupt than $\mathrm{Co} \mid \mathrm{Pt}$, owing to Py's intrinsic disorder. SOC-induced interface splittings are smeared out by alloy disorder in Py compared to Co, leading to smaller $\delta$. Finally, we found that proximityinduced magnetization of Pt by Co or Py has no effect on the interface parameters within the error bars of the calculations; see the left inset to Fig. 3(a).

Summary.-First-principles scattering theory and a recently developed local current scheme have been used to study how spin currents propagate through interfaces between two nonmagnetic ( $\mathrm{Au} \mid \mathrm{Pt}$ and $\mathrm{Au} \mid \mathrm{Pd})$ materials and between a ferromagnetic and a nonmagnetic (Py|Pt and $\mathrm{Co} \mid \mathrm{Pt})$ material at finite temperatures. By extracting values of $\delta, R_{\mathrm{I}}$, and $\gamma$, we could study how $\delta$ depends on various properties of the interfaces and temperature. For nonmagnetic interfaces, we found that $\delta$ and $A R_{\mathrm{I}}$ remain unchanged over a wide range of temperature and found values of $A R_{\mathrm{I}}$ that are in remarkably good agreement [50] with an ansatz introduced more than twenty years ago by Schep et al [10].

$\delta_{\mathrm{Au} \mid \mathrm{Pt}}$ was found to be larger than $\delta_{\mathrm{Au} \mid \mathrm{Pd}}$ owing to the larger SOC in Pt, indicating a direct link between the magnitude of $\delta$ and SOC strength of NM metals. An incommensurable $\mathrm{Au} \mid \mathrm{Pt}$ interface with relaxed $\mathrm{Au}$ and $\mathrm{Pt}$ lattices has a substantially larger $\delta$ than the lattice-matched interface. Mixing at an interface also leads to larger values of $\delta$. Thus, to minimize $\delta$, lattice-matched and clean interfaces should be targeted in experiments to avoid momentumnonconserving scattering of conduction electrons.

$\mathrm{FM} \mid \mathrm{Pt}$ interface parameters decrease strongly with increasing temperature. This dependence stems directly from the magnetization of the FM. Co is a stronger FM than $\mathrm{Py}$ and we find that $A R_{\mathrm{Co} \mid \mathrm{Pt}}$ and $\delta_{\mathrm{Co} \mid \mathrm{Pt}}$ are larger than $A R_{\mathrm{Py} \mid \mathrm{Pt}}$ and $\delta_{\mathrm{Py} \mid \mathrm{Pt}}$ for all temperatures. By turning off spin disorder in $\mathrm{Py} \mid \mathrm{Pt}$, the variation of interface parameters with temperature becomes negligible. 
This work was financially supported by the "Nederlandse Organisatie voor Wetenschappelijk Onderzoek" (NWO) through the research program of the former "Stichting voor Fundamenteel Onderzoek der Materie," (NWO-I, formerly FOM) and through the use of supercomputer facilities of NWO "Exacte Wetenschappen" (Physical Sciences). K. G. acknowledges funding from the Shell-NWO/FOM Computational Sciences for Energy Research Ph.D. program (CSER-PhD; No. i32; Project No. 13CSER059) and is grateful to Yi Liu for help in starting this work and to S. Wildeman for helpful discussions. The work was also supported by the Royal Netherlands Academy of Arts and Sciences (KNAW). Work in Beijing was supported by the National Natural Science Foundation of China (Grant No. 61774018), the Recruitment Program of Global Youth Experts, and the Fundamental Research Funds for the Central Universities (Grant No. 2018EYT03).

*zyuan@bnu.edu.cn

†P.J.Kelly@utwente.nl

[1] M. N. Baibich, J. M. Broto, A. Fert, F. Nguyen Van Dau, F. Petroff, P. Etienne, G. Creuzet, A. Friederich, and J. Chazelas, Giant Magnetoresistance of (001)Fe/(001)Cr Magnetic Superlattices, Phys. Rev. Lett. 61, 2472 (1988).

[2] G. Binasch, P. Grünberg, F. Saurenbach, and W. Zinn, Enhanced magnetoresistance in layered magnetic structures with antiferromagnetic interlayer exchange, Phys. Rev. B 39, 4828 (1989).

[3] P. C. van Son, H. van Kempen, and P. Wyder, Boundary Resistance of the Ferromagnetic-Nonferromagnetic Metal Interface, Phys. Rev. Lett. 58, 2271 (1987).

[4] T. Valet and A. Fert, Theory of the perpendicular magnetoresistance in magnetic multilayers, Phys. Rev. B 48, 7099 (1993).

[5] J. Bass, CPP magnetoresistance of magnetic multilayers: A critical review, J. Magn. Magn. Mater. 408, 244 (2016).

[6] J. Bass and W. P. Pratt, Jr., Spin-diffusion lengths in metals and alloys, and spin-flipping at metal/metal interfaces: An experimentalist's critical review, J. Phys. Condens. Matter 19, 183201 (2007).

[7] J. Sinova, S. O. Valenzuela, J. Wunderlich, C. H. Back, and T. Jungwirth, Spin Hall effects, Rev. Mod. Phys. 87, 1213 (2015).

[8] R. J. H. Wesselink, K. Gupta, Z. Yuan, and P. J. Kelly, Calculating spin transport properties from first principles: Spin currents, Phys. Rev. B 99, 144409 (2019).

[9] C. Galinon, K. Tewolde, R. Loloee, W.-C. Chiang, S. Olson, H. Kurt, W. P. Pratt, Jr., J. Bass, P. X. Xu, K. Xia, and M. Talanana, $\mathrm{Pd} / \mathrm{Ag}$ and $\mathrm{Pd} / \mathrm{Au}$ interface specific resistances and interfacial spin flipping, Appl. Phys. Lett. 86, 182502 (2005).

[10] K. M. Schep, Jeroen B. A. N. van Hoof, P. J. Kelly, G. E. W. Bauer, and J. E. Inglesfield, Interface resistances of magnetic multilayers, Phys. Rev. B 56, 10805 (1997).

[11] M. D. Stiles and D. R. Penn, Calculation of spin-dependent interface resistance, Phys. Rev. B 61, 3200 (2000).
[12] K. Xia, P. J. Kelly, G. E. W. Bauer, I. Turek, J. Kudrnovský, and V. Drchal, Interface resistance of disordered magnetic multilayers, Phys. Rev. B 63, 064407 (2001).

[13] P. X. Xu, K. Xia, M. Zwierzycki, M. Talanana, and P. J. Kelly, Orientation-Dependent Transparency of Metallic Interfaces, Phys. Rev. Lett. 96, 176602 (2006).

[14] K. D. Belashchenko, A. A. Kovalev, and M. van Schilfgaarde, Theory of Spin Loss at Metallic Interfaces, Phys. Rev. Lett. 117, 207204 (2016).

[15] K. Dolui and B. K. Nikolić, Spin-memory loss due to spin-orbit coupling at ferromagnet/heavy-metal interfaces: $A b$ initio spin-density matrix approach, Phys. Rev. B 96, 220403(R) (2017).

[16] A. Hoffmann, Spin Hall effects in metals, IEEE Trans. Magn. 49, 5172 (2013).

[17] M. I. Dyakonov and V.I. Perel, Current-induced spin orientation of electrons in semiconductors, Phys. Lett. 35A, 459 (1971).

[18] J. E. Hirsch, Spin Hall Effect, Phys. Rev. Lett. 83, 1834 (1999).

[19] S. Zhang, Spin Hall Effect in the Presence of Spin Diffusion, Phys. Rev. Lett. 85, 393 (2000).

[20] J.-C. Rojas-Sánchez, N. Reyren, P. Laczkowski, W. Savero, J.-P. Attané, C. Deranlot, M. Jamet, J.-M. George, L. Vila, and H. Jaffrès, Spin Pumping and Inverse Spin Hall Effect in Platinum: The Essential Role of Spin-Memory Loss at Metallic Interfaces, Phys. Rev. Lett. 112, 106602 (2014).

[21] E. Saitoh, M. Ueda, H. Miyajima, and G. Tatara, Conversion of spin current into charge current at room temperature: Inverse spin-Hall effect, Appl. Phys. Lett. 88, 182509 (2006).

[22] K. Ando, S. Takahashi, K. Harii, K. Sasage, J. Ieda, S. Maekawa, and E. Saitoh, Electric Manipulation of Spin Relaxation Using the Spin Hall Effect, Phys. Rev. Lett. 101, 036601 (2008).

[23] O. Mosendz, J. E. Pearson, F. Y. Fradin, G. E. W. Bauer, S. D. Bader, and A. Hoffmann, Quantifying Spin Hall Angles from Spin Pumping: Experiments and Theory, Phys. Rev. Lett. 104, 046601 (2010).

[24] O. Mosendz, V. Vlaminck, J. E. Pearson, F. Y. Fradin, G. E. W. Bauer, S. D. Bader, and A. Hoffmann, Detection and quantification of inverse spin Hall effect from spin pumping in permalloy/normal metal bilayers, Phys. Rev. B 82, 214403 (2010).

[25] L. Liu, T. Moriyama, D. C. Ralph, and R. A. Buhrman, Spin-Torque Ferromagnetic Resonance Induced by the Spin Hall Effect, Phys. Rev. Lett. 106, 036601 (2011).

[26] T. Kimura, Y. Otani, T. Sato, S. Takahashi, and S. Maekawa, Room-Temperature Reversible Spin Hall Effect, Phys. Rev. Lett. 98, 156601 (2007).

[27] L. Vila, T. Kimura, and Y. Otani, Evolution of the Spin Hall Effect in Pt Nanowires: Size and Temperature Effects, Phys. Rev. Lett. 99, 226604 (2007).

[28] Y. Liu, Z. Yuan, R. J. H. Wesselink, A. A. Starikov, and P. J. Kelly, Interface Enhancement of Gilbert Damping from First Principles, Phys. Rev. Lett. 113, 207202 (2014).

[29] M.-H. Nguyen, D. C. Ralph, and R. A. Buhrman, Spin Torque Study of the Spin Hall Conductivity and Spin Diffusion Length in Platinum Thin Films with Varying Resistivity, Phys. Rev. Lett. 116, 126601 (2016). 
[30] E. Sagasta, Y. Omori, M. Isasa, M. Gradhand, L. E. Hueso, Y. Niimi, Y. Otani, and F. Casanova, Tuning the spin Hall effect of Pt from the moderately dirty to the superclean regime, Phys. Rev. B 94, 060412(R) (2016).

[31] L. Wang, R. J. H. Wesselink, Y. Liu, Z. Yuan, K. Xia, and P. J. Kelly, Giant Room Temperature Interface Spin Hall and Inverse Spin Hall Effects, Phys. Rev. Lett. 116, 196602 (2016).

[32] V. P. Amin and M. D. Stiles, Spin transport at interfaces with spin-orbit coupling: Formalism, Phys. Rev. B 94, 104419 (2016).

[33] L. J. Zhu, D. C. Ralph, and R. A. Buhrman, Irrelevance of magnetic proximity effect to spin-orbit torques in heavymetal/ferromagnet bilayers, Phys. Rev. B 98, 134406 (2018).

[34] L. Zhu, D. C. Ralph, and R. A. Buhrman, Spin-Orbit Torques in Heavy-Metal Ferromagnet Bilayers with Varying Strengths of Interfacial Spin-Orbit Coupling, Phys. Rev. Lett. 122, 077201 (2019).

[35] A. J. Berger, E. R. J. Edwards, H. T. Nembach, O. Karis, M. Weiler, and T. J. Silva, Determination of the spin Hall effect and the spin diffusion length of $\mathrm{Pt}$ from self-consistent fitting of damping enhancement and inverse spin-orbit torque measurements, Phys. Rev. B 98, 024402 (2018).

[36] L. Zhu, D. C. Ralph, and R. A. Buhrman, Enhancement of spin transparency by interfacial alloying, Phys. Rev. B 99, 180404(R) (2019).

[37] L. Zhu, D. C. Ralph, and R. A. Buhrman, Effective SpinMixing Conductance of Heavy-Metal Ferromagnet Interfaces, Phys. Rev. Lett. 123, 057203 (2019).

[38] A. A. Starikov, Y. Liu, Z. Yuan, and P. J. Kelly, Calculating the transport properties of magnetic materials from firstprinciples including thermal and alloy disorder, noncollinearity and spin-orbit coupling, Phys. Rev. B 97, 214415 (2018).

[39] Y. Liu, A. A. Starikov, Z. Yuan, and P. J. Kelly, Firstprinciples calculations of magnetization relaxation in pure $\mathrm{Fe}, \mathrm{Co}$, and $\mathrm{Ni}$ with frozen thermal lattice disorder, Phys. Rev. B 84, 014412 (2011).

[40] Y. Liu, Z. Yuan, R. J. H. Wesselink, A. A. Starikov, M. van Schilfgaarde, and P. J. Kelly, Direct method for calculating temperature-dependent transport properties, Phys. Rev. B 91, 220405(R) (2015).

[41] The results we will describe are valid in the linear response regime that should be accessible using nonlocal spininjection experiments. When properties are determined in switching experiments, i.e., from spin pumping and ISHE measurements or from SHE and SOT measurements, care must be taken to ensure that nonlinear effects can be neglected [52,53].

[42] W. Zhang, W. Han, X. Jiang, S.-H. Yang, and S. S. P. Parkin, Role of transparency of platinum-ferromagnet interfaces in determining the intrinsic magnitude of the spin Hall effect, Nat. Phys. 11, 496 (2015).

[43] D. V. Baxter, S. D. Steenwyk, J. Bass, and W. P. Pratt, Jr., Resistance and spin-direction memory loss at $\mathrm{Nb} / \mathrm{Cu}$ interfaces, J. Appl. Phys. 85, 4545 (1999).

[44] K. Eid, D. Portner, J. A. Borchers, R. Loloee, M. A. Darwish, M. Tsoi, R. D. Slater, K. V. O'Donovan, H. Kurt, W. P. Pratt, Jr., and J. Bass, Absence of mean-free-path effects in the current-perpendicular-to-plane magnetoresistance of magnetic multilayers, Phys. Rev. B 65, 054424 (2002).

[45] CRC Handbook of Chemistry and Physics, 90th Edition (Internet Version 2010), edited by D. R. Lide, 90th ed. (CRC Press/Taylor and Francis, Boca Raton, FL, 2009).

[46] The spin current flowing in the $z$ direction and polarized along the global quantization axis of the system is defined in terms of the nonconserved spin as $j_{s}(z) \equiv$ $\hat{z} \cdot\langle\sigma \otimes \mathbf{v}+\mathbf{v} \otimes \sigma\rangle / 2$. It is normalized with respect to the charge current density and is averaged over the $x y$ plane perpendicular to the transport direction. For more details, see Ref. [8].

[47] M. Isasa, E. Villamor, L. E. Hueso, M. Gradhand, and F. Casanova, Temperature dependence of spin diffusion length and spin Hall angle in Au and Pt, Phys. Rev. B 91, 024402 (2015).

[48] R. Freeman, A. Zholud, Z. Dun, H. Zhou, and S. Urazhdin, Evidence for Dyakonov-Perel-like Spin Relaxation in Pt, Phys. Rev. Lett. 120, 067204 (2018).

[49] Compared to, e.g., $\mathrm{Cu} \mid \mathrm{Pt}$, a distinct advantage of the $\mathrm{Au} \mid \mathrm{Pt}$ interface is that interface mixing can be studied without having to make unreasonable assumptions about the structure of the alloy. There is considerable interest in the spintransport properties of the bulk AuPt [54,55] (AuW [56] and AuTa [57]) alloys, but to do justice to this topic requires a publication in its own right; the present manuscript focuses on interfaces.

[50] Schep et al. expressed the interface resistance in terms of the transmission through an ideal, $T=0 \mathrm{~K}$ interface combined with an ansatz for diffusive scattering in the bulk [10] inspired by random matrix theory. The predictions of this ansatz compare well with experiment as long as the mean free path is shorter than the distance between successive interfaces. Calculating the interface transmission without thermal disorder, with and without SOC, and using Schep's expression for the interface resistance, we find $R_{\mathrm{Au} \mid \mathrm{Pt}}=$ $0.63 \mathrm{f} \Omega \mathrm{m}^{2}$ with SOC and $0.56 \mathrm{f} \Omega \mathrm{m}^{2}$ without, compared to the room-temperature value of $0.54 \pm 0.03 \mathrm{f} \Omega \mathrm{m}^{2}$ that was found above with SOC with the present methodology.

[51] K. Gupta, Disentangling interfaces and bulk in spin transport calculations, Ph. D. thesis, University of Twente, The Netherlands, 2019.

[52] V. E. Demidov, S. Urazhdin, B. Divinskiy, V. D. Bessonov, A. B. Rinkevich, V. V. Ustinov, and S. O. Demokritov, Chemical potential of quasi-equilibrium magnon gas driven by pure spin current, Nat. Commun. 8, 1579 (2017).

[53] I. V. Borisenko, V. E. Demidov, S. Urazhdin, A. B. Rinkevich, and S. O. Demokritov, Relation between unidirectional spin hall magnetoresistance and spin current-driven magnon generation, Appl. Phys. Lett. 113, 062403 (2018).

[54] M. Obstbaum, M. Decker, A. K. Greitner, M. Haertinger, T. N. G. Meier, M. Kronseder, K. Chadova, S. Wimmer, D. Ködderitzsch, H. Ebert, and C. H. Back, Tuning Spin Hall Angles by Alloying, Phys. Rev. Lett. 117, 167204 (2016).

[55] Y. Xu, Y. Yang, H. Xie, and Y. Wu, Spin hall magnetoresistance sensor using $\mathrm{Au}_{x} \mathrm{Pt}_{1-x}$ as the spin-orbit torque biasing layer, Appl. Phys. Lett. 115, 182406 (2019).

[56] P. Laczkowski, J.-C. Rojas-Sánchez, W. Savero-Torres, H. Jaffrès, N. Reyren, C. Deranlot, L. Notin, C.Beigné, 
A. Marty, J.-P. Attané, L. Vila, J.-M. George, and A. Fert, Experimental evidences of a large extrinsic spin Hall effect in AuW alloy, Appl. Phys. Lett. 104, 142403 (2014).

[57] P. Laczkowski, Y. Fu, H. Yang, J.-C. Rojas-Sánchez, P. Noel, V. T. Pham, G. Zahnd, C. Deranlot, S. Collin,
C. Bouard, P. Warin, V. Maurel, M. Chshiev, A. Marty, J.-P. Attané, A. Fert, H. Jaffrès, L. Vila, and J.-M. George, Large enhancement of the spin Hall effect in $\mathrm{Au}$ by side-jump scattering on Ta impurities, Phys. Rev. B 96, 140405(R) (2017). 